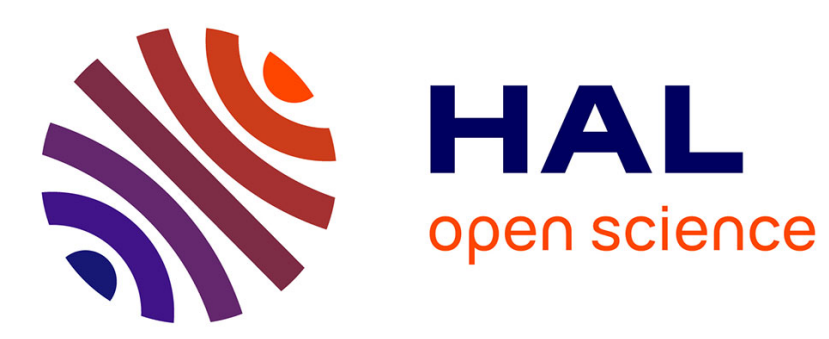

\title{
Hybrid Noise Predictions Of A Radial Notebook Blower
}

\author{
Marlène Sanjosé, Stéphane Moreau, Jessica Gullbrand
}

\section{To cite this version:}

Marlène Sanjosé, Stéphane Moreau, Jessica Gullbrand. Hybrid Noise Predictions Of A Radial Notebook Blower. 16th International Symposium on Transport Phenomena and Dynamics of Rotating Machinery, Apr 2016, Honolulu, United States. hal-01887476

\section{HAL Id: hal-01887476 https://hal.science/hal-01887476}

Submitted on 4 Oct 2018

HAL is a multi-disciplinary open access archive for the deposit and dissemination of scientific research documents, whether they are published or not. The documents may come from teaching and research institutions in France or abroad, or from public or private research centers.
L'archive ouverte pluridisciplinaire HAL, est destinée au dépôt et à la diffusion de documents scientifiques de niveau recherche, publiés ou non, émanant des établissements d'enseignement et de recherche français ou étrangers, des laboratoires publics ou privés. 


\title{
Hybrid Noise Predictions Of A Radial Notebook Blower
}

\author{
Marlene Sanjose $^{1 *}$, Stephane Moreau ${ }^{1}$, Jessica Gullbrand ${ }^{2}$
}

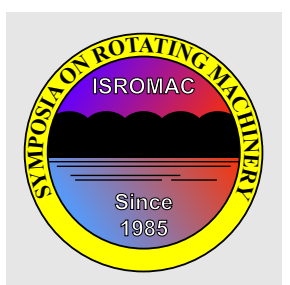

ISROMAC 2016

International

Symposium on

Transport

Phenomena and

Dynamics of

Rotating Machinery

Hawaii, Honolulu

April 10-15, 2016

\section{Abstract}

The tonal noise of a notebook radial blower in a free-field environment is investigated using an hybrid method based on unsteady Reynolds Averaged Navier-Stokes simulations and an Ffowcs Williams and Hawking's analogy to compute the acoustic far-field from the wall-pressure fluctuations recorded in the simulations. Incompressible and compressible simulations have been performed to demonstrate the effect of the compressibility on the noise sources in the very constrained environment due to the casing specific design. The complex flow in the blower yields distributed noise sources. The tongue interaction with the blade wakes and the inlet flow distortions are major contributors at the blade passing frequency, while smaller structures in the clearance are important noise sources at higher harmonics. The influence of a daisy obstruction on one of the blower inlet is also investigated. The additional inlet distortion strongly affects the noise levels at the blade passing frequency. The acoustic predictions are compared to experimental measurements and provide good agreement within the variability of the acoustic level measured between blowers of the same conception batch. The compressible simulations provide the best agreement at higher frequency when the compactness limit is overpassed.

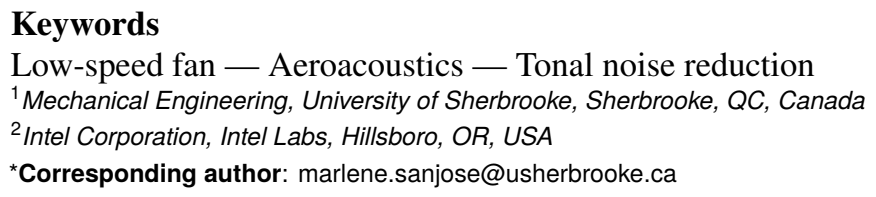

\section{INTRODUCTION}

Small low-speed radial blowers are used in many cooling applications including very compact notebook systems. The noise level produced by these systems is one of the main concerns for customers to not exceed acoustic ergonomic comfort. Thus, aero-acoustic predictions are of great importance for the computer manufacturers at the design level. Notebook blower systems often yield annoying tonal noise. Aero-acoustic sources are multiple in such dense and complex computer systems. Broadband noise component is produced by a wide range of turbulent excitations, while tonal noise component is related to large coherent flow structures strongly depending on the installation. Several measurements have been achieved on small radial blowers meant for cooling notebooks and are described in a previous study by Gullbrand \& Beltman [1, 2]. In the present work, unsteady incompressible and compressible numerical simulations are performed on one standard size blower to predict tonal noise components in free flow conditions. Very few numerical tools allow to capture, at affordable computational cost, both the tonal and broadband sources and the far-field propagation, since it requires to capture large structures related to the installation to very small turbulent structures that appear in boundary layers, and a wide range of wave-numbers on a large computational domain [3, 4]. The present methodology adapted for the tonal noise prediction relies on a hybrid methodology using incompressible and compressible unsteady Reynolds-Averaged Navier-Stokes (URANS) simulations linked with an acoustic propagation tool. The selected flow solver is ANSYS-CFX and it is coupled with an in-house code, SherFWH, applying the Ffowcs Williams and Hawkings acoustic analogy on rotating surfaces.

The present numerical simulations have the following objectives. First they are meant to create a representative set-up on a limited domain that can reliably reproduce the large structures of the flow in free-field, and consequently reproduce the measured tonal noise. Secondly, by comparing incompressible and compressible flow simulations, they evaluate the effect of compressibility on the tonal noise radiation in such low-speed blowers. Finally, they should prove if they can capture noise mitigation effects from daisy obstructions correctly. A side objective is also to test the unstructured mesh strategy for such applications.

\section{EXPERIMENTAL AND NUMERICAL SETUPS}


Figure 1. Notebook blower.

The notebook blower investigated shown in Fig. 1 has standard outer dimensions of $60 \mathrm{~mm} \times 54 \mathrm{~mm} \times 9 \mathrm{~mm}$. The fan is integrated into a fitted casing with two facing axial entrances with circular section and a single radial exit with a rectangular section. One of the inlet is obstructed by three motor struts. In the present investigation the blower is installed 
in free-field as shown in Fig. 2.

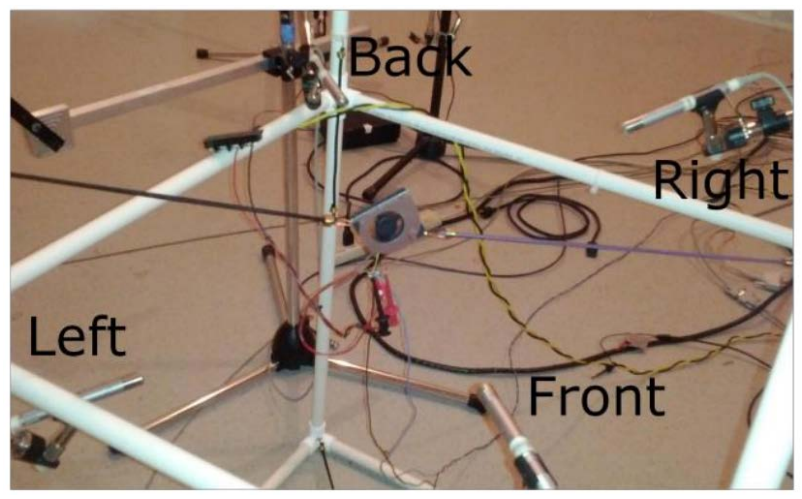

Figure 2. Notebook blower experimental setup.

The fan is located at $900 \mathrm{~mm}$ from the semi-anechoic room reverberant floor. The measurements of acoustic pressure are made at $250 \mathrm{~mm}$ from the rotor center at four locations : two microphones are facing each other along the rotor axis, and two microphones are in the casing plane. In the present study, the rotation speed is $4500 \mathrm{RPM}$.

To investigate passive noise control on this configuration, a daisy obstruction placed at the blower inlet in a similar way as Magne et al. did [5]. The metal sheet from the casing is replaced by a plastic plate with an interchangeable inlet geometry which angular position around the fan axis can be controlled as shown in Fig. 3. Acoustic measurements with similar conditions than performed by Gullbrand \& Beltman $[1,2]$ have been performed in the anechoic room of the Sherbrooke's University. Several daisy shaped inlet with various lobe numbers and lobe amplitude were tested. In the present work, measurements for a daisy obstruction of lobe amplitude $\Delta R=0.4 \mathrm{~mm}$ and lobe number equals the blade number will be presented.

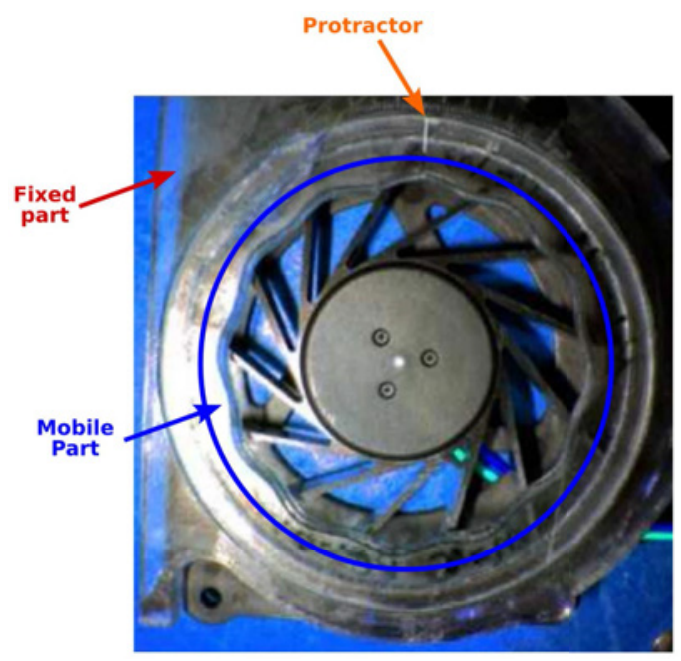

Figure 3. Experimental device for controlling the angular location of the daisy obstruction.



(a) Computational domain.

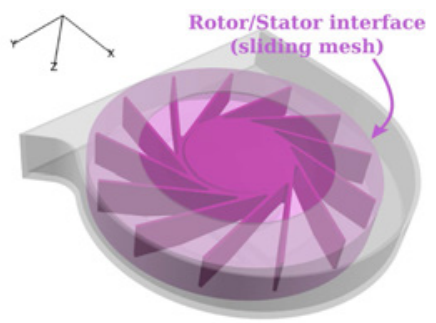

(b) Rotor-stator interface.
Figure 4. CFX numerical set-up.

The numerical set-up used in the present study is shown in Fig. 4. The blower is placed in the middle of a large sphere of $150 \mathrm{~mm}$ radius. As outlined in the zoom on the blower, the interface between the rotating and stationary part is placed at mid-distance from the casing closest position. The fan has 13 blades made of long flat plates, allowing the flow from both entrances, and strongly inclined from the radial direction.

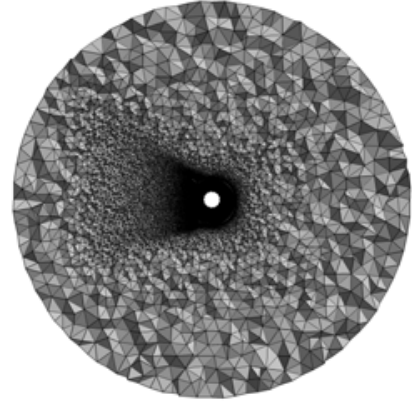

(a) Global mesh view.



(b) Detail view of the tongue area.
Figure 5. Unstructured mesh views.

A mesh of tetrahedrons is generated using the CentaurSoft mesh generator. The final mesh size with overall dimensionless wall distance $y^{+} \approx 5$ has 3 million nodes and 17 million tetrahedral elements. As can be seen in Fig. 5 the cells are clustered in the casing and in the wake development area. The same unstructured grid methodology as was used for a larger radial fan has been applied here [6]. The same clustering in the tongue and casing regions have been considered. Only tetrahedrons have been considered however due to the particular blade connections to the hub, that would have yield strong prismatic cell chopping and inappropriate mesh quality.

The Mach number based on the tip velocity is lower than 0.04, so compressibility effects are negligeable. Four different simulations have been run and are summarized in Tab. 1. The influence of using a compressible solver and adapted non-reflective characteristic boundary conditions (NSCBC) is investigated [7]. While such a solver should not modify the flow due to the low Mach number of the simulation, it may have an effect on the noise sources on the diffracting elements. The first three simulations consider the baseline configuration. The last one adds the daisy obstruction placed at the blower 
Table 1. Simulations of the low-speed radial blower investigated in the present study.

\begin{tabular}{lcc} 
& Solved equation & Configuration \\
\hline CFX - I0 & incompressible & Baseline \\
\hline CFX - C0 & compressible & Baseline \\
\hline CFX - C1 & compressible + NSCBC & Baseline \\
\hline CFX - I0D & incompressible & Obstruction \\
\hline
\end{tabular}

inlet. The daisy of 13 lobes with amplitude $\Delta R=0.4 \mathrm{~mm}$ was tested with an arbitrary angular position $\Delta \theta=2.5^{\circ}$ as shown in Fig. 6.



Figure 6. Modified inlet of the configuration with the daisy obstruction replacing the circular inlet shown with the red line.

All unsteady simulations use the sliding mesh method and a $k-\omega$ SST model for turbulence. The simulations are initialized from a prior RANS simulation using a frozen-method at the interface. The unsteady simulations use the high resolution scheme from CFX which is second order in space and 33 time-steps are performed per blade passage period, while twenty sub-iterations are performed per time-step. After about one revolution of the rotor, the torque reaches a stabilized state for which it oscillates around a constant value.

\section{PERFORMANCES AND FLOW FIELD}

\subsection{Global Performances}

The performances obtained for the four numerical simulations are reported in Tab. 2 . The numerical results provide volume flow-rate within 1 to $2 \%$ of the measured flow-rate for the baseline configuration. The obstruction yields no noticeable effect on the computed flow rate. This was later verified experimentally by comparing the heat dissipation with and without daisy obstruction.

\subsection{Analysis of the flow field}

The flow structures for the $C F X-I O$ and $C F X-C 1$ are very similar and described in this section. The overall flow of the blower is shown in Fig. 7. The streamlines coming from the inlet are colored in red, while the streamlines coming from the inlet with motor struts are colored in blue. The outlet flow is split into two jets. The main jet is on the opposite side from the casing tongue. The main jet is stratified, the blue lines coming from the upper inlet being mainly on the upper part of
Table 2. Fan system performances at free flow.

\begin{tabular}{lcc} 
Case & Flow rate & Torque \\
\hline Experiments & $8.86 \mathrm{~m}^{3} \mathrm{~h}^{-1}$ & - \\
\hline CFX - I0 & $8.85 \mathrm{~m}^{3} \mathrm{~h}^{-1}$ & $6.52 \times 10^{-4} \mathrm{~N} \mathrm{~m}$ \\
\hline CFX - C0 & $8.75 \mathrm{~m}^{3} \mathrm{~h}^{-1}$ & $6.56 \times 10^{-4} \mathrm{~N} \mathrm{~m}$ \\
\hline CFX - C1 & $8.79 \mathrm{~m}^{3} \mathrm{~h}^{-1}$ & $6.48 \times 10^{-4} \mathrm{~N} \mathrm{~m}$ \\
\hline CFX - I0D & $8.80 \mathrm{~m}^{3} \mathrm{~h}^{-1}$ & $6.50 \times 10^{-4} \mathrm{~N} \mathrm{~m}$ \\
\hline
\end{tabular}

the jet. The secondary jet structures are more turbulent with wavy streamlines and more mixing between the blue and red lines coming from the two inlets.

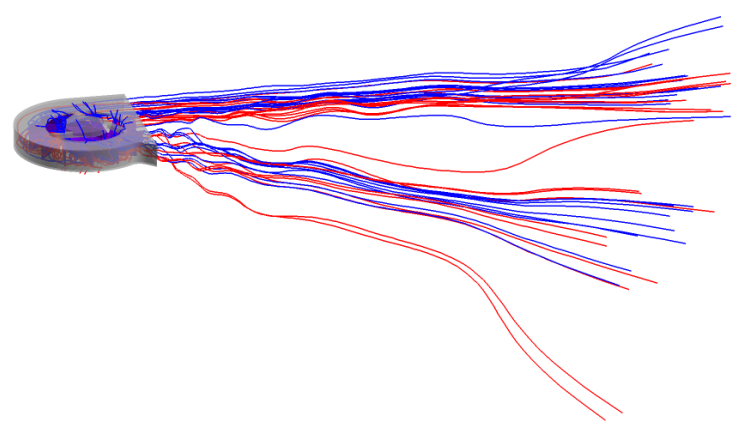

Figure 7. Streamlines of an instantaneous field for the baseline configuration.

In the coordinate basis used in the setup, the exit face from the casing is pointing towards negative $\mathrm{x}$-direction. A typical instantaneous axial velocity field is shown in Fig. 8. The two jet structures observed in Fig. 7 cover the full thickness of the blower. The main jet stream has larger velocity and a larger width than the jet on the tongue side. This is clearly related to the fan installation in the casing shown in Fig. 4(b). In between, recirculation areas can be observed. In the middle of the casing thickness the flow is exiting, while some flow is coming back towards the fan on the upper and lower side.

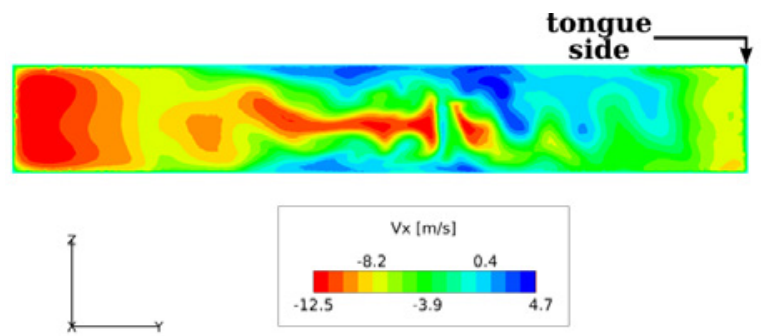

Figure 8. Instantaneous velocity map at the casing exit

The flow in the blower is quite complex as shown in Fig. 9 on the mid-section plane. The back of the casing is a zone of high pressure and low velocity. This zone drives the main jet stream observed previously. The complex blade wakes with strong vortices close to the casing exit explain the recirculation areas observed in Fig. 8. The interactions of the blade wakes 
with the tongue generate pockets of high pressure oscillations, that are efficient noise sources for the casing.

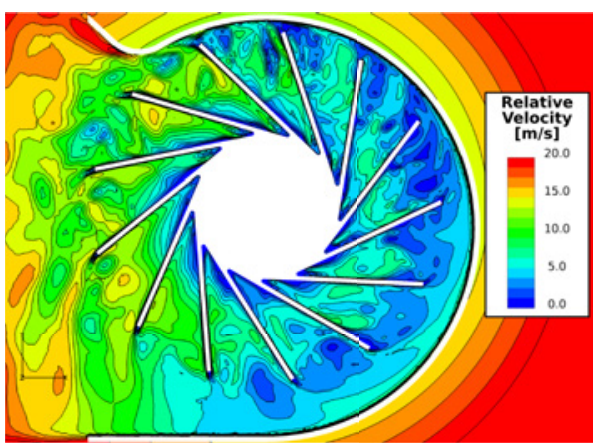

(a) Relative velocity.

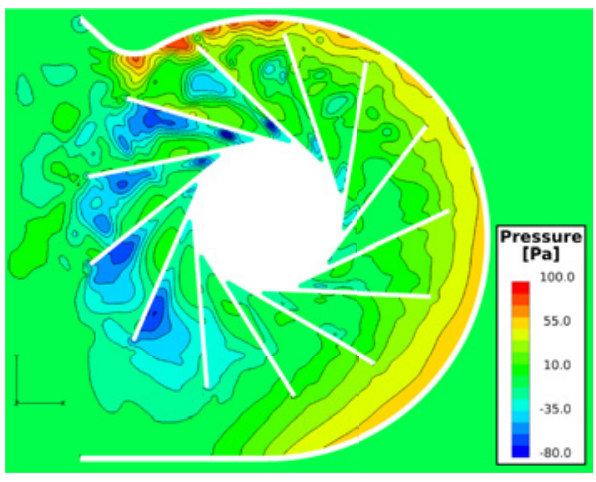

(b) Pressure

Figure 9. Instantaneous field at mid-section of the casing.

The inlet distortion is responsible for the noise sources on the fan surfaces. It can be identified using the Q-criterion (the second-invariant of velocity gradient tensor) iso-surfaces as shown in Fig. 10. The inlet velocity in the stationary frame is shown for each inlet sides. The instantaneous velocity distortions seen on the circular inlet are related to both the rotor potential effect and the pressure distribution seen in Fig. 9. The suction is more favorable on the front side leading to flow detachment on half of the circular inlet. On the back side of the casing some structures are visible, they are related to strong velocity unsteadiness seen in Fig.9. They create unsteady blockage for the inlet flow but are rotating with the fan. On the motor side, the velocity distortions are stronger. The structures are related to the motor struts. The swirling inflow sheds vortices in the wake of the back strut that are sucked into the casing. These structures are stationary in the absolute reference and will directly contribute to the tonal noise sources on the fan surfaces.

\subsection{Influence of the characteristic boundary con- ditions}

The two compressible simulations yield similar flow features in the blower, but the pressure field in the computational domain is strongly affected by the boundary condition on the outlet atmospheric patch. Using the standard boundary condition for the compressible simulation yields an unphysical



(a) Clean circular inlet.

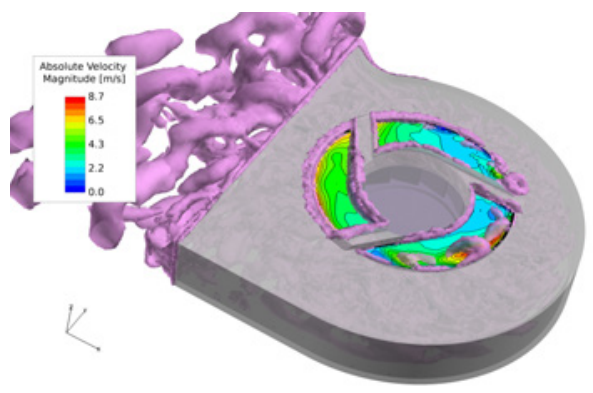

(b) Motor side inlet.

Figure 10. Instantaneous flow at inlet sections.

pressure distribution in the computational domain caused by strong reflection on the outlet sphere as shown in Fig. 11. The pressure fluctuations on the rotor and casing for the simulation $C F X$ - $C O$ generate unphysical acoustic predictions $40 \mathrm{~dB}$ higher than $C F X-C l$ predictions.
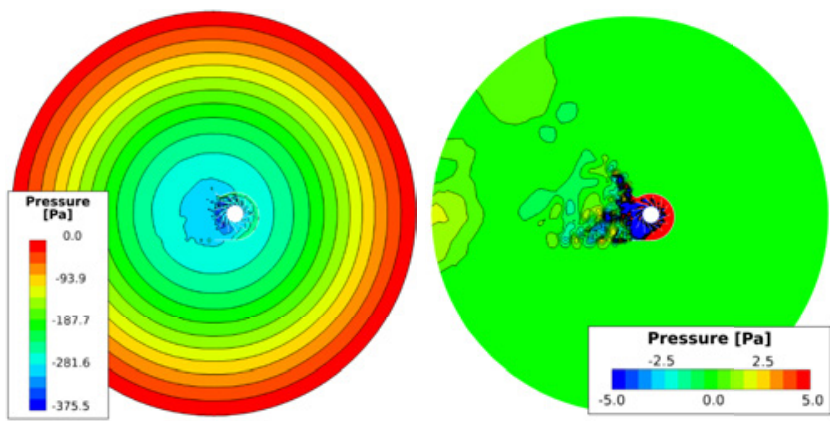

(a) Standard boundary conditions (b) Non-reflective boundary condiCFX-CO. tions $C F X-C 1$

Figure 11. Instantaneous pressure field at mid-section.

\subsection{Influence of the daisy obstruction}

The circular inlet is modified by the insertion of the daisy obstruction. The inlet section area is the same as in the baseline case. Still this slight modulation of the inlet modifies the inlet distortion as shown in Fig. 12. The rotating structures observed in Fig. 10 in the back of the casing on the circular inlet appear on a larger part of the inlet and influence more the inner part of the inflow. They are more coherent and they interact between each other while they were only attached to 
the rotating blade in the baseline case. To quantify this effect, the absolute velocity is extracted on a $2 \mathrm{~mm}$ inner circle from the inlet plane. On this particular path, the circumferential mean of the absolute velocity increases by $6 \%$ in the daisy case compared with the baseline case and the standard deviation increases from $30 \%$ to $35 \%$ of the circumferential mean. The daisy obstruction yields an increased distortion velocity and becomes an additional tonal noise source on the fan. On the opposite side, no major difference can be observed.

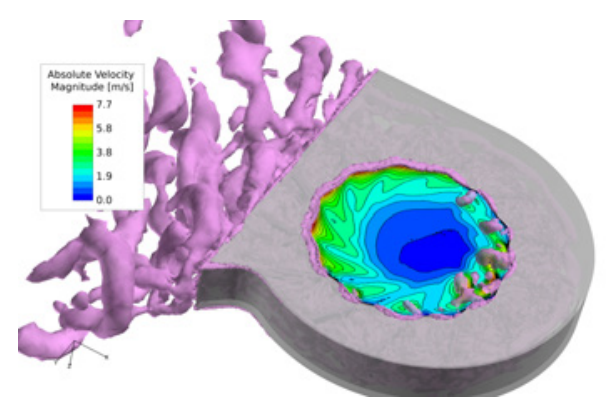

(a) Daisy inlet.

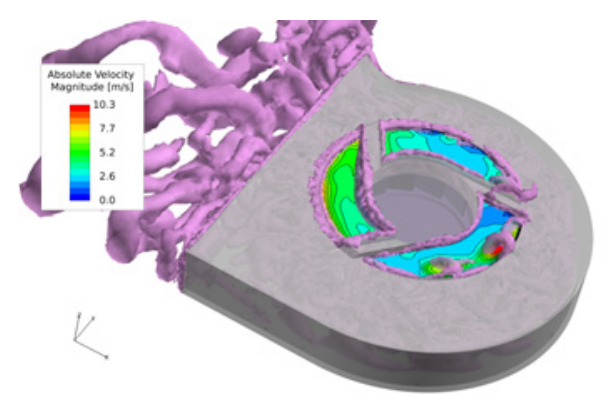

(b) Motor side inlet.

Figure 12. Instantaneous flow at inlet sections.

\section{ACOUSTIC SOURCES}

The wall pressure fluctuations are recorded on the fan and casing surfaces along the simulations. The spectral density distribution for the first three harmonics is shown in Fig. 13. The zones with intense density are the main noise sources contributing to the far-field acoustic. The sources are dominant on the inner wall of the casing. At the BPF, the tongue is a strong contributor, while for the higher harmonics the most intense fluctuations appear close to the inlet where the inflow sees a strong deviation and is affected by the rotating blades. On the fan, the stronger sources are located at the tip of the blade close to the casing wall on the motor side where the inlet distortions observed in Fig. 10 impact the blades.

\section{ACOUSTIC PREDICTIONS}

The acoustic sources are the wall pressure fluctuations on the casing and the rotor. This is a reasonable assumption since the maximum blower Mach number is below 0.04. These fluctuations are then fed to a Ffowcs Williams and Hawking's analogy model as implemented in the in-house tool

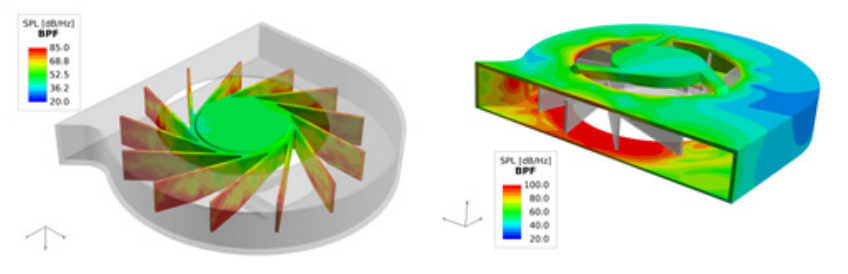

(a) BPF

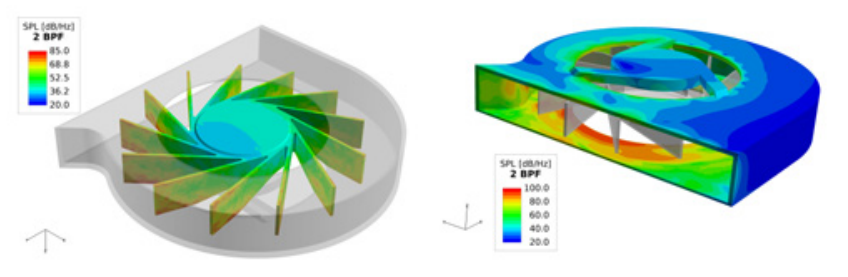

(b) $2 \mathrm{BPF}$

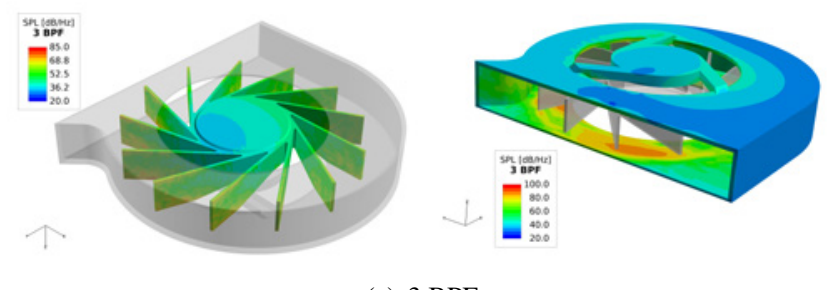

(c) $3 \mathrm{BPF}$

Figure 13. Wall pressure density spectra.

SherFWH $[8,9]$. Three different blowers (termed Blower 1, Blower 2, Blower 3) were used in the experiments, and the results show the effects of blower-to-blower variability [2]. No significant difference is seen on the broadband noise component below $4 \mathrm{kHz}$ with increasing differences on Blower 1 beyond this frequency. Much more variations are found on the tones obtained with the three blowers: up to $6.5 \mathrm{~dB}$ differences on the first and second harmonics and up to $10 \mathrm{~dB}$ on the third harmonics.

\subsection{Time convergence}

The incompressible simulation $C F X-I O$ is run for $0.1 \mathrm{~s}$. The compressible simulation $C F X-C l$ being more expensive in terms of CPU resources, the convergence of the acoustic was verified during the simulation. The acoustic results obtained from the Ffowcs Williams and Hawking's analogy based on noise sources collected during $0.03 \mathrm{~s}$ and $0.1 \mathrm{~s}$ are shown in Fig. 14 for the microphone facing the clean inlet. For the shorter source recording time, the predicted levels for the tones are about $10 \mathrm{~dB}$ to $15 \mathrm{~dB}$ higher than the longer source recording time. Only the fundamental frequency is not affected. The respective distribution from the casing and the rotor are not affected. The increased simulation time allows better capturing the rotor/stator interaction and the non-compact source interferences at the microphone location. 


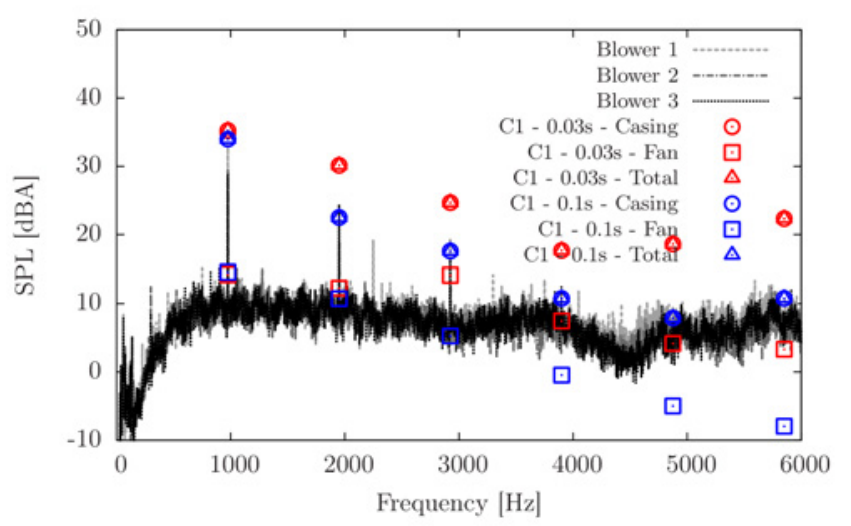

Figure 14. Noise spectra comparisons between experiments and $C F X-C 1$ simulations (red: $T_{\text {sim }}=0.03 \mathrm{~s}$ and blue: $\left.T_{\text {sim }}=0.1 \mathrm{~s}\right)$.

\subsection{Incompressible and compressible noise sources}

Figure 15 presents the acoustic results obtained from the Ffowcs Williams and Hawking's analogy based on noise sources collected during $0.1 \mathrm{~s}$ for the incompressible $C F X-I O$ and compressible simulation $C F X-C 1$. The sound pressure level measured on both sides of the blower are compared with measurements performed by Gullbrand \& Beltman [1, 2]. The first two harmonics yield good agreement between the experiments and the simulations (both incompressible and compressible simulations). Only the compressible simulation yield satisfactory levels up to $5 \mathrm{kHz}$. Around $4 \mathrm{kHz}$ the compactness limit is reached. The Ffowcs Williams and Hawking's analogy is adapted to free-field propagation, only the compressible simulation can account for the multiple acoustic reflexions in the casing on the pressure fluctuations that increase the levels. Similar observations were made on isolated airfoils [10] and low-speed fan using noise sources from incompressible or compressible simulations $[6,11,12,13]$. The main contribution comes from the casing in agreement with the higher wall-pressure fluctuations observed on the casing in Fig. 13, while the fan tonal noise sources do not emerge from the broadband noise level.

\subsection{Acoustic effect of the daisy obstruction}

To evaluate the experimental setup at Université de Sherbrooke (UdeS) and the influence of the casing modification for the daisy obstruction installation shown in Fig. 3, a circular inlet equivalent to the original design inlet was tested. The measurements for the microphone facing the modified UdeS inlet shown in Fig. 16 with the red dashed line are compared with the measurements from Gullbrand \& Beltman [1, 2] shown with black and grey lines. Good agreement is again found up to $4 \mathrm{kHz}$ with larger discrepancies at high frequencies. This first comparison thus demonstrates the equivalence of the two test installations and that the casing modification has no significant effect on the acoustic spectra except at



(a) Microphone facing the clean inlet.



(b) Microphone facing the motor.

Figure 15. Noise spectra comparisons between experiments and simulations (red: incompressible and blue: compressible).

very high frequencies where some local diffraction can be expected. The acoustic measurement with the daisy obstruction for a position of $\Delta \theta=2.5^{\circ}$ (blue dashed line) show a quasi-identical broadband noise level but significantly modified tonal noise. It is compared for the same microphone facing the modified inlet with the acoustic results obtained from the Ffowcs Williams and Hawking's analogy based on noise sources collected during $0.1 \mathrm{~s}$ for the incompressible CFX-IO simulation of the baseline and for the incompressible $C F X-I O D$ simulation of the modified case. Similar results are obtained for other microphone positions.

Because the lobe number of the obstruction equals the blade number, the first tone is strongly affected by the inlet modification both numerically and experimentally. For this particular position the tone level increases of $15 \mathrm{~dB}$ in the numerical measurements and $6 \mathrm{~dB}$ in the experiments. The second harmonics is reduced by $2 \mathrm{~dB}$ and $3 \mathrm{~dB}$ for the numerical predictions and measurements respectively. For the third and above harmonics the noise levels are increased by $2 \mathrm{~dB}$ to 
$5 \mathrm{~dB}$.

The obstruction not only changes the fan noise contribution as could have been predicted from the modification of the inlet distortion shown in Fig. 12, but it also affects the casing sources. The system is so compact, that it is difficult to add an isolated source that can be phased-lag to the main noise source and bring noise reduction.



Figure 16. Noise spectra comparisons between experiments and simulations (red: baseline and blue: obstruction).

In the experimental investigation the angular position of the daisy obstruction has been varied. At each location the noise spectra for the microphone facing the daisy obstruction is recorded. The levels of the first two tones is shown as a function of the angular position in Fig. 17. As expected, the first harmonics is strongly influenced by the daisy obstruction angular position. An effective noise reduction is observed for a particular angle $\Delta \theta=12.5^{\circ}$, while amplification is measured for most of the angles. The second harmonics is less affected by the daisy position, a slight reduction if observed in most of the cases. Finding the best angular position with numerical methods is quite resources consuming and is not performed in the present work. An original methodology involving a slowly counter rotating obstruction was proposed and validated by Magne [14].

\section{CONCLUSIONS}

The tonal noise of a typical computer blower system has been successfully predicted using an hybrid approach based on a URANS simulation with ANSYS CFX and the Ffowcs Williams and Hawking's analogy to propagate the acoustic in the far-field. The influence of the fluid model has been evaluated by comparing results from a incompressible and a compressible simulation. The boundary conditions must be non-reflective when using a compressible solver to capture physical noise sources within the simulation. The flow in the blower system is quite complex, due to the two opposite inlet and the closeness of the casing from the rotor blade trailingedge. A two-jet exit-flow structure is observed, created by the pressure recovery from the casing and by the interaction of

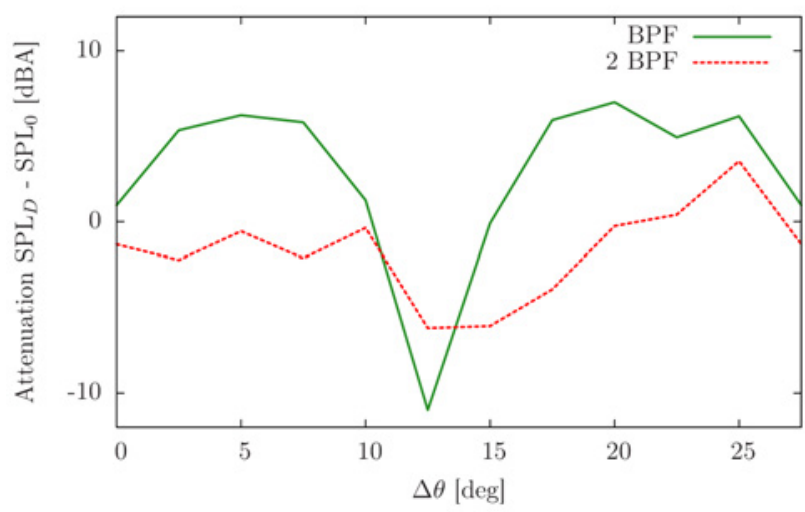

Figure 17. Experimental noise reduction for the first two harmonics as a function of the daisy angular position.

the rotor blade with the volute tongue. The main sources appear on the casing due to the impact of the rotor wakes. Inlet distortions are sources on the fan surfaces. They are created by the motor struts and the pressure field in the casing. One of the inlet is modified by inserting a periodic daisy-obstruction pattern. It affects the inlet distortions on that side. The acoustic predictions are compared with measurements, and provide good agreement in the range of variability observed between three identical blower systems. The compressible simulations provide a better agreement at high frequency whereas the incompressible simulation is only accurate up to the compactness limit of the fan as found previously on airfoils [10] and axial fans [11]. The main contributions come from the casing sources as identified with the spectral density of the wallpressure fluctuations. The inlet modification with the daisy obstruction affects strongly the first harmonics of both the casing and the fan contributions demonstrating that the tonal noise system could be controlled by such a passive device. An optimization of the lobe amplitude and its angular position could be performed either experimentally or numerically following the methodology developed in [14].

\section{ACKNOWLEDGMENTS}

Computations were made on the supercomputer MammothMP2 from Université de Sherbrooke, managed by Calcul Québec and Compute Canada. The operation of this supercomputer is funded by the CFI, NanoQuébec, RMGA and FRQ-NT. The authors would like to acknowledge NSERC for funding this INNOV research project with the participation of Intel as industrial partner.

\section{REFERENCES}

[1] J. Gullbrand and W.M. Beltman. Numerical and experimental acoustic analysis of small scale radial notebook blower. In Inter-Noise 2012, August 2012. 
[2] J. Gullbrand and W.M. Beltman. Experiments And Numerical Predictions Of Flow Rates And Aero-Acoustics From Small Radial Notebook Blowers . In Fan 2012, April 2012.

[3] T. Carolus, M. Schneider, and H. Reese. Axial flow fan broad-band noise and prediction. J. Sound Vib., 300(12):50-70, 2007.

[4] F. Perot, M.-S. Kim, S. Moreau, and M. Henner. Direct aeroacoustics prediction of a low speed axial fan. In $16^{\text {th }}$ AIAA/CEAS Aeroacoust. Conf., AIAA-2010-3887, Stockholm, Sweden, June 2010.

[5] S. Magne, M. Sanjose, S. Moreau, and A. Berry. Numerical Optimization of Fan Tonal Noise Control using Acoustic Modulation of Slowly-Rotating Obstructions. In 20 ${ }^{\text {th }}$ AIAA/CEAS Aeroacoust. Conf., AIAA-2014-2948, June 2014.

[6] M. Sanjose and S. Moreau. Numerical simulations of a low-speed radial fan. Int. J. Eng. Syst. Model. Sim., 4(1-2):47-58, 2012.

[7] T. Poinsot and S.K. Lele. Boundary conditions for direct simulations of compressible viscous flows. J. Comp. Phys., 101(1):104-129, 1992.

[8] A. Fosso Pouangué, M. Sanjosé, S. Moreau, G. Daviller, and H. Deniau. Subsonic jet noise simulations using both structured and unstructured grids. AIAA J., 53(1):55-69, 2015.

[9] A. Marsan and S. Moreau. Aeroacoustic analysis of the tonal noise of a large-scale radial blower. In ISROMAC16, $16^{\text {th }}$ Symposium on Transport Phenomena and Dynamics of Rotating Machinery, April 2016.

[10] J. Christophe, J. Antoine, and S. Moreau. Trailing-edge noise of a controlled-diffusion airfoil at oderate and high angle of attack. In $15^{\text {th }}$ AIAA/CEAS Aeroacoust. Conf., AIAA-2009-3196, May 2009.

[11] M. Sanjose, S. Magne, and S. Moreau. Aeroacoustic predictions of a low-speed radial fan. In ISROMAC-15, $15^{\text {th }}$ Symposium on Transport Phenomena and Dynamics of Rotating Machinery, February 2014.

[12] S. Magne, S. Moreau, and A. Berry. Subharmonic tonal noise from backflow vortices radiated by a low-speed ring fan in uniform inlet flow. J. Acoust. Society Am., 137(1), January 2015.

[13] M. Sanjose, D. Lallier-Daniels, and S. Moreau. Aeroacoustic Analysis Of A Low-Subsonic Axial Fan. In Proceedings of ASME Turbo Expo 2015: Turbine Technical Conference $\mathcal{F}$ Exposition, GT2015-43737, June 2015.

[14] S. Magne. Approche numérique du contrôle du bruit tonal des ventilateurs par obstruction de l'écoulement. $\mathrm{PhD}$ thesis, Université de Sherbrooke, October 2015. 